\title{
Characteristics and outcomes of therapy-related myeloid neoplasms following autologous stem cell transplantation for multiple myeloma
}

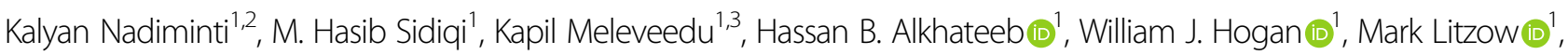

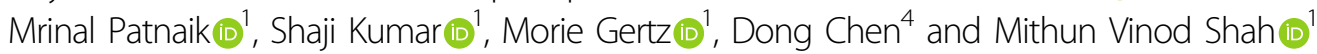

\section{Dear Editor,}

Advances over the last two decades have resulted in a significant improvement in survival of patients with multiple myeloma (MM) - primarily driven by the emergence of immunomodulators (IMiDs), proteasome inhibitors (PI) and monoclonal antibodies. Despite these improvements, high-dose melphalan (HDM)-based autologous stem cell transplantation (SCT) followed by lenalidomide maintenance remains part of standard initial therapy for transplant-eligible patients ${ }^{1}$. The development of therapyrelated myeloid neoplasms ( $t-\mathrm{MN})$ is an uncommon, but dreadful complication with considerable morbidity and poor survival. While the exposure to melphalan and autologous SCT are known risk factors ${ }^{2,3}$, the impact of lenalidomide, in the setting of HDM, is less clear ${ }^{4-7}$. Autologous SCT followed by lenalidomide maintenance leads to an improved progression-free- and overall survi$\mathrm{val}$, making it the de facto standard for eligible patients ${ }^{5,6}$. We studied the incidence of $t-M N$ in post-SCT MM patients in the era of novel therapies and studied the impact of lenalidomide exposure on the risk of $\mathrm{t}-\mathrm{MN}$ postSCT. We also analyzed factors that may impact outcomes after $\mathrm{t}-\mathrm{MN}$ diagnosis.

We identified MM patients that underwent autologous SCT following HDM (1998-2016) and subsequently developed t-MN as defined by the 2016 World Health Organization classification. Complex and monosomal karyotype (CK and MK respectively) were defined according to the 1995 International System for Human Cytogenetic

\footnotetext{
Correspondence: Mithun Vinod Shah (shah.mithun@mayo.edu)

${ }^{1}$ Division of Hematology, Mayo Clinic, Rochester, MN, USA

${ }^{2}$ Division of Hematology, Oncology, and Palliative Care, University of

Wisconsin, Madison, WI, USA

Full list of author information is available at the end of the article
}

Nomenclature recommendations. Myeloid mutational analysis was obtained using the clinically available 43-gene next-generation sequencing (NGS) panel available at Mayo Clinic, Rochester. Overall survival (OS) was calculated using the standard Kaplan-Meier technique. Univariate and multivariate analyses using Cox proportional hazard model were performed to study the interaction of risk factors with the outcomes. Statistical analyses were performed using SAS (JMP v14.1) and GraphPad Prism (v7). Factors purported to predispose to $\mathrm{t}-\mathrm{MN}$ development such as alkylator therapy including HDM, a second autologous SCT, and exposure to lenalidomide were analyzed. Outcome variables were assessed from the time of $\mathrm{t}-\mathrm{MN}$ diagnosis, except for the cumulative incidence of $\mathrm{t}-\mathrm{MN}$, which was assessed from the time of MM diagnosis.

We found 2448 consecutive patients that underwent first autologous SCT for MM. Of these, 52 patients (2.1\%) developed t-MN. Thirty-seven of 52 (71\%) patients with $\mathrm{t}-\mathrm{MN}$ had received lenalidomide. In contrast, of 2396 patients that did not develop t-MN, 1285 (54\%) had received lenalidomide. Thus, lenalidomide exposure was associated a significantly higher risk of $\mathrm{t}-\mathrm{MN}\left(\chi^{2}\right.$ with Yate's correction 5.6, $P=0.002$, Supplementary Fig. 1). Our analysis is in agreement with a recent report of $\mathrm{t}-\mathrm{MN}$ in post-SCT patients that post-SCT lenalidomide was associated with a higher risk of $\mathrm{t}-\mathrm{MN}$ and all patients that developed $\mathrm{t}-\mathrm{MN}$ had been on lenalidomide or thalidomide $^{7}$. To account for lenalidomide-associated survival bias, a time-dependent analysis of the incidence of $t-M N$ was performed. There was a significant increase in the incidence of $\mathrm{t}-\mathrm{MN}$ among the patients that received lenalidomide compared to those who did not $(P<0.0001$, Supplementary Fig. 2). Patients that developed t-MN had a higher median number of apheresis sessions compared to 
Table 1 Clinical and pathologic characteristics of multiple myeloma (MM) patients that developed therapy-related myeloid neoplasm (t-MN).

\begin{tabular}{|c|c|c|}
\hline Characteristics & $\mathrm{t}-\mathrm{MDS}(n=36)$ & $\mathrm{t}-\mathrm{AML}(n=7)$ \\
\hline Age at MM diagnosis (years), median (range) & $61(41-74)$ & $58(47-73)$ \\
\hline Age at autologous SCT (years), median (range) & $64.5(41-74)$ & $59(48-73)$ \\
\hline Age at t-MN diagnosis (years), median (range) & $70(44-79)$ & $61(51-77)$ \\
\hline Sex, male (\%) & $25(70 \%)$ & $6(85 \%)$ \\
\hline Time from autologous SCT to t-MN (years), median (range) & $5(1-15)$ & $4(1-9)$ \\
\hline Transformed to AML, (\%) & $13(36 \%)$ & $\mathrm{n} / \mathrm{a}$ \\
\hline Time to transform to AML (months), median (range) & $6(3-17)$ & $\mathrm{n} / \mathrm{a}$ \\
\hline Patients that underwent second SCT for MM, $n(\%)$ & $6(16 \%)$ & $1(14 \%)$ \\
\hline Patients that received lenalidomide pre-SCT, $n(\%)$ & $9(21 \%)$ & $3(43 \%)$ \\
\hline Patients that received lenalidomide post-SCT, $n(\%)$ & $28(66 \%)$ & $6(85 \%)$ \\
\hline Patients that received lenalidomide $\geq 2$ years post-SCT, $n(\%)$ & $12(33 \%)$ & $4(57 \%)$ \\
\hline Cycles of chemotherapy containing alkylators, including HDM and cyclophosphamide for mobilization, median (range) & $1(0-6)$ & $1(1-4)$ \\
\hline Proportion with blast $\% \geq 10, n(\%)$ & $6(17 \%)$ & $\mathrm{n} / \mathrm{a}$ \\
\hline Complex karyotype, $n(\%)$ & $18(50 \%)$ & $5(72 \%)$ \\
\hline Monosomal karyotype, $n(\%)$ & $25(70 \%)$ & $4(57 \%)$ \\
\hline Complex and monosomal karyotype, $n(\%)$ & $13(36 \%)$ & $4(57 \%)$ \\
\hline Received hypomethylating agents for t-MN, $n$ (\%) & $13(38 \%)$ & $1(16 \%)$ \\
\hline Allogeneic SCT for t-MN, $n$ (\%) & $3(8 \%)$ & $1(14 \%)$ \\
\hline Autologous SCT for t-MN, $n$ (\%) & $4(11 \%)$ & None \\
\hline Overall survival in months since t-MN, median ( $95 \% \mathrm{Cl}$ ) & $15(10-18)$ & $6(2-14)$ \\
\hline
\end{tabular}

$t$-MDS therapy-related myelodysplastic syndrome, $t$-AML therapy-related acute myeloid leukemia, MM multiple myeloma, SCT stem cell transplant, $H D M$ high dose melphalan, $t-M N$ therapy-related myeloid neoplasm.

those that did not develop t-MN (3 vs. 2, $P=0.0007$, Supplementary Fig. 3), while the number of median CD34 ${ }^{+}$ cells collected did not differ ( 8.8 vs. 9.2, $P=0.5)$. Exposure to alkylators [melphalan, cyclophosphamide, low-dose melphalan, $\mathrm{BCNU}]$ and anthracycline (AC, yes vs. no) did not predict the development of $\mathrm{t}-\mathrm{MN}$ (data not shown).

Nine patients were excluded due to lack of follow-up or unavailability of clinical data. Baseline characteristics of 43 patients shown in Table 1 . Thirty-one (72\%) were males and the median age at diagnosis was 70 years (range 44-79) for t-MDS and 61 years (range 51-77) for t-AML. Median time from autologous SCT to t-MN development for the entire cohort was 5 years (range 1-15) while it was 5 years (range 1-15) for MDS and 4 years (range 1-9) for AML. Seven patients (16\%) presented with t-AML, and 36 (84\%) with t-MDS. Pathologic and treatment features are tabulated (Supplementary Table 1). Of the 36 patients with t-MDS, 13 (36\%) transformed to AML during their clinical course with the median time to transformation being 6 months (range 3-17). MK and CK were predominant, accounting for $51 \%$ each in t-MDS, and $57 \%$ and $72 \%$ of patients with $\mathrm{t}$-AML, respectively. NGS at the time of
t-MN diagnosis was available for $13(30 \%)$ patients, of which 7 (50\%) had mutated TP53.

Initial treatment of $\mathrm{t}-\mathrm{MN}$ included hypomethylating agents (HMA), lenalidomide, induction chemotherapy, or supportive care alone. Fourteen (32.5\%) patients received only supportive care either due to poor performance status or due to a low-risk disease. Of the remaining 29 (67.5\%), $13(36 \%)$ and $1(14 \%)$ received HMA as the frontline therapy among $\mathrm{t}$-MDS and $\mathrm{t}$-AML groups, respectively. Five $(71.5 \%)$ patients with $\mathrm{t}$-AML and $3(8 \%)$ with $\mathrm{t}$-MDS received induction chemotherapy and $6(75 \%)$ required salvage therapy. Overall, 8 (19\%) patients proceeded to receive an autologous SCT from previously collected PBSC $(n=4)$ or allogeneic $(n=4)$ transplant, of which only one patient was from t-AML group (Supplementary Table 2). The rest $(21,48 \%)$ did not proceed to SCT due to inadequate control of the disease.

After a median follow-up of 70 months [95\% confidence interval (CI), 38-134], the median OS was 12 months (95\% CI, 9-17, Fig. 1). There was no difference in survival for those with t-MDS or t-AML (15 vs. 6 months, $P=0.2$ ), those who underwent the transplant, compared to those 


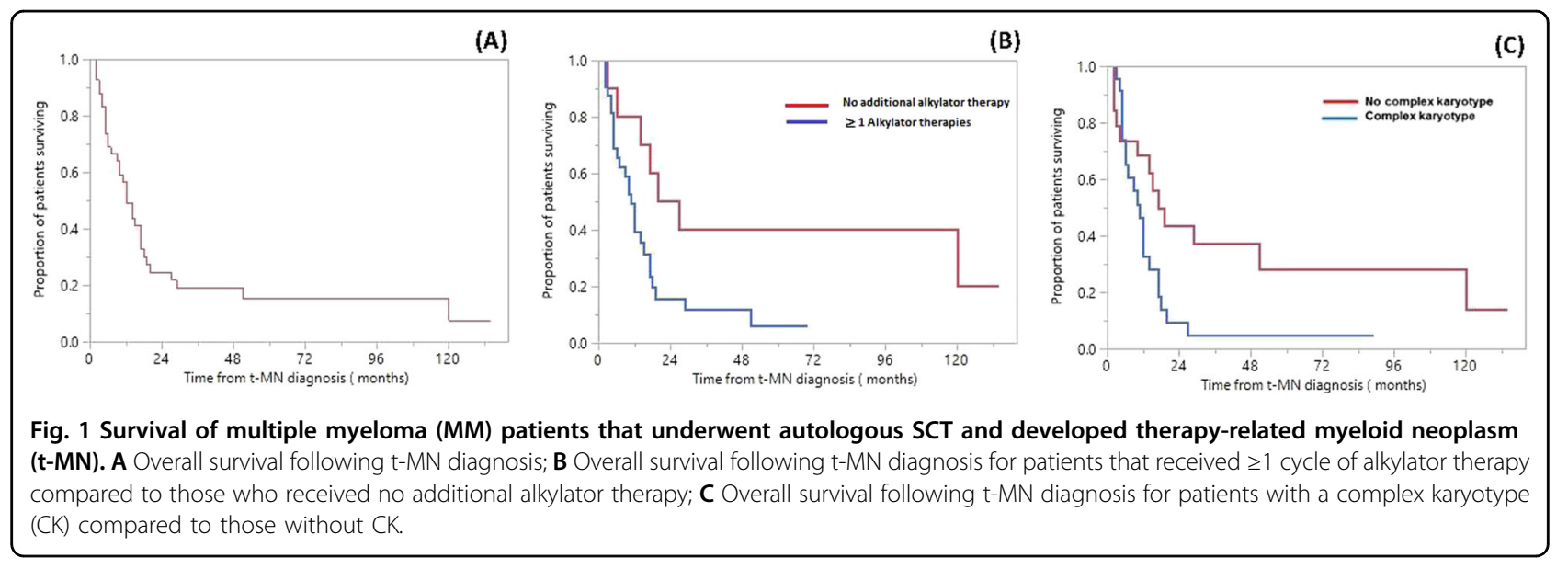

who did not (17 vs. 10 months, $P=0.3$ ), or those who received allo SCT compared to autologous SCT (21 vs. 16 months, $P=0.7$ ). At the time of the last follow-up, 9 (21\%) patients were alive. Primary causes of death were progressive $\mathrm{t}-\mathrm{MN}(71 \%), \mathrm{MM}(12 \%)$, or both $(6 \%)$. Among the eight patients that underwent any $\mathrm{SCT}$, one person was alive at the last follow-up. Five (62.5\%) patients died of $\mathrm{t}-\mathrm{MN}$, one each died of progressive $\mathrm{MM}$, and transplanted-related mortality (Supplementary Table 2).

We then ascertained the association of cumulative exposure to alkylator therapy with $\mathrm{t}-\mathrm{MN}$ outcomes. Median number of alkylator/AC therapy cycles used prior to autologous SCT was 1 (range 0-6) among both t-MDS and t-AML groups. Seven (17\%) patients received two autologous SCTs. On univariable analysis, factors that predicted $\mathrm{OS}$ following $\mathrm{t}-\mathrm{MN}$ diagnosis were exposure to $\geq 1$ line of alkylator therapies 11 vs. 27 months, $(P=0.02)$, the presence of $\geq 10 \%$ vs. $<10 \%$ blast at the time of $\mathrm{t}-\mathrm{MN}$ diagnosis (5.5 vs. 17 months, $P=0.01$ ), and the presence of CK vs. not (11 vs. 17 months, $P=0.03$ ). There was no difference in survival among t-MDS patients who transformed to t-AML vs. those who did not, those who received 1 vs. 2 autologous SCT, and those with lenalidomide exposure duration $\geq 2$ vs. $<2$ years. On multivariable analysis, the number of lines of alkylator therapies, the presence of CK, and the total \% blasts remained as significant predictors of OS (Supplementary Table 3).

A unique aspect of our study is the availability of patientspecific disease and treatment characteristics that provide a better insight to the natural history of $\mathrm{t}-\mathrm{MN}$. In a large cohort of patients that received HDM and autologous SCT for MM, we observed a 5.6-fold increase in the risk of $\mathrm{t}-\mathrm{MN}$ in patients receiving lenalidomide compared to those who did not, which is in agreement with a recent study $^{7}$. Our results are consistent with the published experience of cumulative $\mathrm{t}-\mathrm{MN}$ incidence ranging between $0.5-6 \%$ in the era of autologous SCT with HDM and extended use of novel agents, including IMiDs, in MM.
t-MN is a well-recognized complication occurring following exposure to cytotoxic chemotherapy and radiotherapy, including in those who receive autologous SCT. Complex karyotype and TP53 mutations are very common across $\mathrm{t}-\mathrm{MN}$ and portend a poor response to therapy and dismal survival, as we observed ${ }^{3,8}$. We found t-MDS as a more common initial presentation, as reported previously $^{8,9}$ - with nearly one-third transforming to $\mathrm{t}$-AML eventually. We observed phenotypes of acute erythroid leukemia in three and megakaryoblastic leukemia in one patient respectively, which are known to be frequently associated with CK, TP53 mutation and a dismal survival ${ }^{10}$. At the same time, about $16 \%$ of our patients had stable disease without treatment, indicating the heterogeneity of this entity. In patients that required therapy, the response to conventional AML therapies was poor as most patients required salvage therapies. Ultimately, we noted only two long-term survivors, arbitrarily defined as those who survived $\geq 5$ years-one each following autologous and allogeneic SCT. This is consistent with prior reports in $\mathrm{t}-\mathrm{MN}$ showing marginal benefit of autologous SCT with around 20\% long-term survival, owing to preponderance of CK and TP53 mutations ${ }^{9,11}$.

Limitations of our study include those of it being a retrospective, an observational study spanning 2 decades. Since our cohort consisted of patients undergoing autologous SCT, we were unable to identify the impact of HDM on t-MN development. Cumulative exposure to alkylators, the duration of lenalidomide therapy, and radiation were not consistently available for patients that did not develop t-MN, precluding analyses of their impact on t-MN development. Finally, the unavailability of NGS data for a majority of patients limited our capacity to evaluate the role of genetic factors in transplant outcomes.

Nonetheless, our results emphasize the cumulative risk of alkylators along with lenalidomide in the development of $\mathrm{t}$ $\mathrm{MN}^{4}$. Whether the development of t-MN is strictly due to exposures such as chemotherapy or radiation is controversial. Patients with monoclonal gammopathy of 
unclear significance as well as those with MM that have not received MM-directed therapy, are at a higher risk of developing myeloid neoplasms ${ }^{12,13}$. Phenotypic abnormalities reminiscent of myelodysplastic syndrome (MDS-PA), present in up to $11 \%$ of MM patients at diagnosis ${ }^{14}$; and clonal hematopoiesis $(\mathrm{CH})$, present in $21 \%$ patients undergoing $\mathrm{SCT}^{7}$ have been studied as potential biomarkers for $\mathrm{t}-\mathrm{MN}$. However, neither MDS-PA nor $\mathrm{CH}$ are predictive of $\mathrm{t}-\mathrm{MN}$. As conventional therapies for $\mathrm{t}-\mathrm{MN}$ remain ineffective, there is an urgent need to identify factors that predict the development of t-MN and survival thereafter-our study is a step in that direction.

\section{Author details}

${ }^{1}$ Division of Hematology, Mayo Clinic, Rochester, MN, USA. ${ }^{2}$ Division of Hematology, Oncology, and Palliative Care, University of Wisconsin, Madison, WI, USA. ${ }^{3}$ Division of Division of Bone Marrow Transplantation, Roger Williams Medical Center, Providence, RI, USA. ${ }^{4}$ Division of Hematopathology, Mayo Clinic, Rochester, MN, USA

\section{Author contributions}

K.N. and M.V.S. designed the study. K.N., M.H.S., K.M., S.K., and M.V.S. collected and analyzed data. H.B.A., W.J.H., M.P., S.K., D.C., and M.V.S. interpreted data and wrote the manuscript.

\section{Conflict of interest}

The authors declare no competing interests.

\section{Publisher's note}

Springer Nature remains neutral with regard to jurisdictional claims in published maps and institutional affiliations.

Supplementary information The online version contains supplementary material available at https://doi.org/10.1038/s41408-021-00454-y.

Received: 3 December 2020 Revised: 23 February 2021 Accepted: 4 March 2021

Published online: 19 March 2021

\section{References}

1. Kumar, S. K. Buadi, F. K. \& Rajkumar, S. V. Pros and cons of frontline autologous transplant in multiple myeloma: the debate over timing. Blood 133, 652-659 (2019).

2. Benboubker, L. et al. Lenalidomide and dexamethasone in transplantineligible patients with myeloma. N. Engl. J. Med. 371, 906-917 (2014).

3. Radivoyevitch, T. et al. Risk of acute myeloid leukemia and myelodysplastic syndrome after autotransplants for lymphomas and plasma cell myeloma. Leukemia Res. 74, 130-136 (2018).

4. Palumbo, A. et al. Second primary malignancies with lenalidomide therapy for newly diagnosed myeloma: a meta-analysis of individual patient data. Lancet Oncol. 15, 333-342 (2014).

5. McCarthy, P. L. et al. Lenalidomide after stem-cell transplantation for multiple myeloma. N. Engl. J. Med. 366, 1770-1781 (2012).

6. Attal, M. et al. Lenalidomide maintenance after stem-cell transplantation for multiple myeloma. New England J. Med. 366, 1782-1791 (2012).

7. Mouhieddine, T. H. et al. Clonal hematopoiesis is associated with adverse outcomes in multiple myeloma patients undergoing transplant. Nat. Commun. 11, 2996 (2020)

8. Pemmaraju, N. et al. Characteristics and outcomes of patients with multiple myeloma who develop therapy-related myelodysplastic syndrome, chronic myelomonocytic leukemia, or acute myeloid leukemia. Clin. Lymphoma Myeloma Leuk. 15, 110-114 (2015)

9. Gertz, M. A. et al. Therapy-related myelodysplastic syndrome/acute leukemia after multiple myeloma in the era of novel agents. Leuk. Lymphoma $\mathbf{5 6}$ 1723-1726 (2015)

10. Wang, S. A. \& Hasserjian, R. P. Acute erythroleukemias, acute megakaryoblastic leukemias, and reactive mimics: a guide to a number of perplexing entities Am. J. Clin. Pathol. 144, 44-60 (2015).

11. Litzow, M. R. et al. Allogeneic transplantation for therapy-related myelodysplastic syndrome and acute myeloid leukemia. Blood 115, 1850-1857 (2010).

12. Mailankody, S. et al. Risk of acute myeloid leukemia and myelodysplastic syndromes after multiple myeloma and its precursor disease (MGUS). Blood 118, 4086-4092 (2011).

13. Roeker, L. E. et al. Risk of acute leukemia and myelodysplastic syndromes in patients with monoclonal gammopathy of undetermined significance (MGUS): a population-based study of 17315 patients. Leukemia 27, 1391-1393 (2013).

14. Maia, C. et al. Biological and clinical significance of dysplastic hematopoiesis in patients with newly diagnosed multiple myeloma. Blood 135, 2375-2387 (2020). 